\title{
Nature-inspired materials: Emerging trends and prospects
}

\author{
Nirmal Kumar Katiyar ${ }^{1,2}$, Gaurav Goel $\mathbb{D}^{1,2}$, Sara Hawi ${ }^{2}$ and Saurav Goel $\mathbb{B}^{1,2,3}$
}

\begin{abstract}
The term "nature-inspired" is associated with a sequence of efforts to understand, synthesize and imitate any natural object or phenomenon either in a tangible or intangible form, which allows us to obtain improved insights into nature. Such inspirations can come through materials, processes, or designs that we see around us. Materials, as opposed to processes and designs found in nature, are tangible and can readily be used without engineering efforts. One such example is that of an aquaporin that is used to filter water. The scope of this work in nature-inspired materials is to define, clarify, and consolidate our current understanding by reviewing examples from the laboratory to industrial scale to highlight emerging opportunities. A careful analysis of "nature-inspired materials" shows that they possess specific functionality that relies on our ability to harness particular electrical, mechanical, biological, chemical, sustainable, or combined gains.
\end{abstract}

\section{Introduction}

Nature has served mankind as a great source of inspiration by virtue of millions of well-coordinated, engineered, and crafted processes, algorithms, materials, and designs. These days, a wide range of nature-inspired products are available in the niche market, as shown in Fig. 1.

Currently, terms such as bioinspiration, biomimicry, biomimetics, nature inspiration, and nature mimicry are often used synonymously in the literature. In this context, words with "nature" prefixes capture the broad ecosystem of living and nonliving natural systems, and words with "bio" prefixes are associated only with living natural organisms (biology) and are contained within the broad spectrum of nature, as shown in Fig. 2. Therefore, while biomimicry or bioinspiration fall within the terms "Nature mimicry" and "Nature inspiration", respectively, the opposite is not true.

ISO 18458:2015(E), an established international standard on this topic, describes subtle differences in the

\footnotetext{
Correspondence: Saurav Goel (GoeLs@Lsbu.ac.uk)

'School of Engineering, London South Bank University, London SE10 AA, UK ${ }^{2}$ School of Aerospace, Transport and Manufacturing, Cranfield University, Bedford MK43 OAL, UK

Full list of author information is available at the end of the article
}

words bioinspiration, biomimetics, and biomimicry. Taking the learnings from this ISO standard and various other sources, we suggest that the term "inspiration" refers to the primitive stage of observation of a certain design or functionality that stimulates creativity and seeds an idea of developing something similar. "Mimetic" implies a further step that involves the application of technology to engineer/manufacture materials inspired by nature to exploit certain functionality observed in nature. "Mimicry" is the most advanced form of inspiration and involves applying engineering and technological tools to develop materials akin to nature with the prime objective of achieving sustainability. Overall, it appears that while inspiration is a primitive step toward mimicking nature, mimicry is the most advanced form that needs engineering perfection to achieve sustainability, while biomimetics represents an intermittent stage between the two.

Currently, interest is growing toward multifunctional step-up mimetics (bottom-up approach) such as a selfcleaning building, where the building blocks making up the building can be inspired by crystal structures (FCC, BCC, HCP, etc.) to achieve higher strength ${ }^{1}$ and achieve a building architecture resembling the shape of natural 


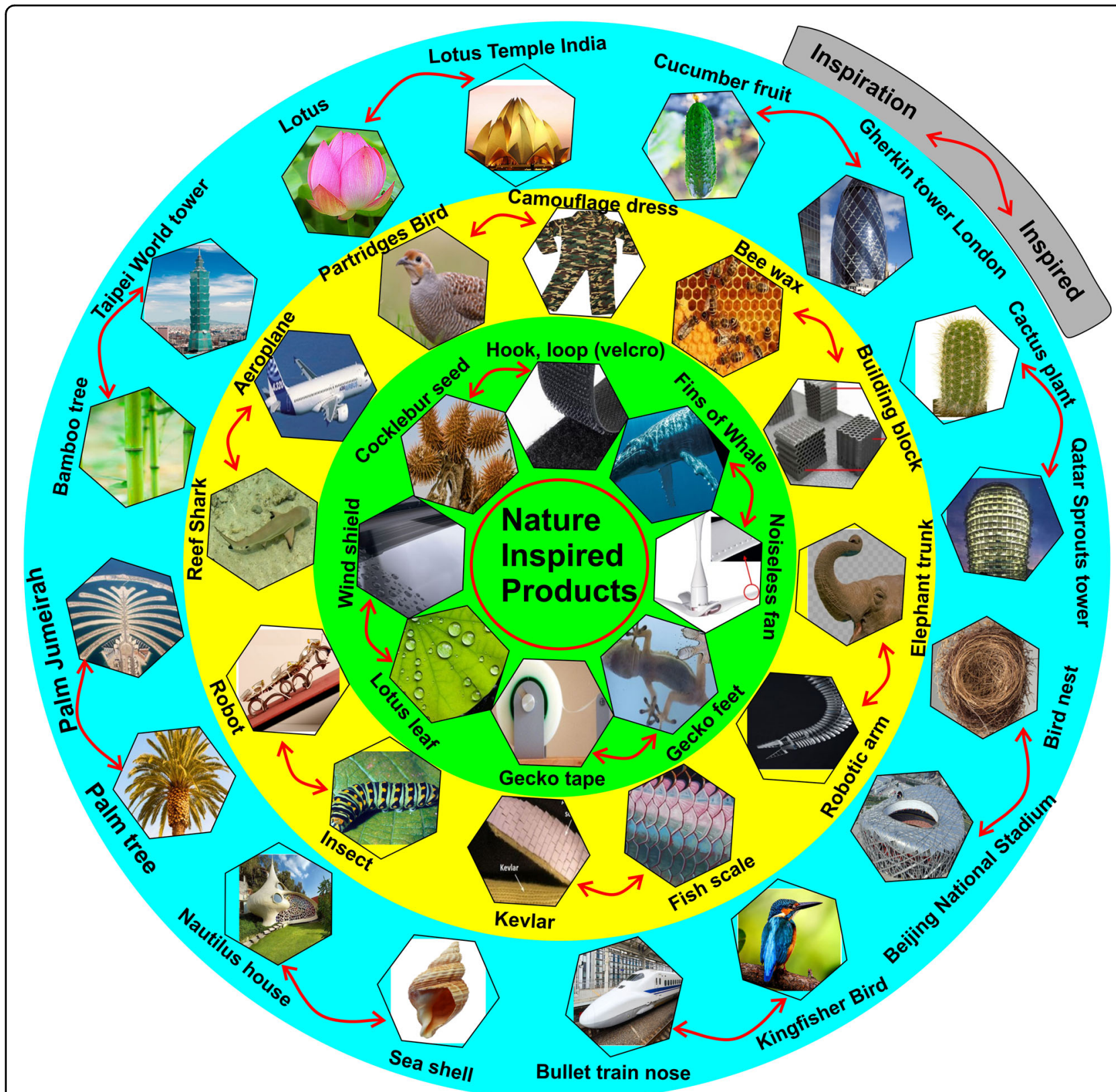

Fig. 1 Commercially available nature-inspired products. Green circles represent functional mimetics, yellow circles represent feature mimetics, and cyan represents world-remarkable architecture inspired by nature.

objects such as a termite mound. This can further be coupled with additional consideration of having the outer wall surface of the same building inspired by a selfcleaning lotus effect ${ }^{2}$ or photosynthesis inspired by a tree ${ }^{3}$, color changes inspired by bird wings or peacock feathers, etc. Such a complex multifunctional and multiscale mimetic (enabling multiple sustainable functionalities) requires a holistic approach, and work in this direction is still in its infancy. The scope of this review is to discuss the latest advances in the field of "nature-inspired materials" in terms of design, manufacturing, and inspirational sources that highlight the current trends.

\section{Broad classification of nature inspiration Nature-inspired processes}

Nature-inspired processes are artificial processes which enable the emulation of a certain natural process such as photosynthesis. An artificial photosynthesis process can therefore be used to harvest solar energy or for solar-tofuel conversion. A nature-inspired process in this case is 


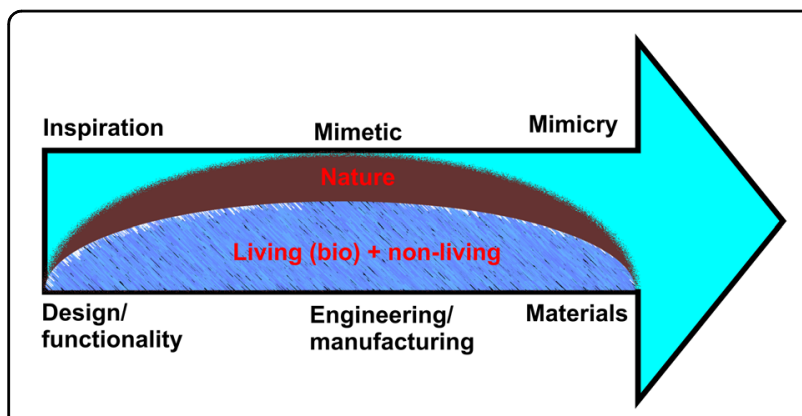

Fig. 2 Classification scheme. Arrow direction shows a generic classification of nature inspiration, mimetics and mimicry.

triggered by the observation of photosynthesis of plant/tree leaves (storing energy in the form of chemical bonds). Recently, many systems have been developed to harvest solar energy, such as a system having flower-like nanostructures generated from copper phosphate nanocomposites, in which $\mathrm{TiO}_{2}$ nanoparticles were incorporated over the petals of a flower (or copper phosphate nanosheets). The copper phosphate flower provides a large surface area to bind $\mathrm{TiO}_{2}$ nanoparticles, whereby the $\mathrm{TiO}_{2}$ nanoparticles act as photocatalysts. Therefore, a copper phosphate flower functionalized with $\mathrm{TiO}_{2}$ nanoparticles works as a solar light harvesting device ${ }^{3,4}$. This system works as an antenna for solar light absorption and splits the water molecules into $\mathrm{O}_{2}$ and $\mathrm{H}_{2}$ (clean energy as a hydrogen fuel cell) gas. This process is akin to photosynthesis in plants. A comparison of natural photosynthesis and nature-inspired artificial photosynthesis is shown in Fig. 3a, b.

A similar strategy has been applied to self-cleaning surfaces (solar panels, walls, etc.), wherein $\mathrm{TiO}_{2}$, as a photocatalyst, was coated over a surface to degrade or split organic dirt photocatalytically into its constituents in the presence of UV light and help water spread over the surface (rinsing the surface) due to hydrophilicity, which allows the surface to become self-cleaned ${ }^{5}$.

Ceramics found in nature utilize less energy and form at mild temperatures. This occurs by a natural process called biomineralization. In contrast, manmade ceramics require higher temperatures above $1400^{\circ} \mathrm{C}$ for densification. The higher temperature densification of the materials is an obstacle because it reduces the material properties due to the coarsening of the grain size. Therefore, adapting a geologically inspired ${ }^{6}$ biomineralization process can produce denser ceramic materials such as calcium carbonate (nanovaterite), which forms during the low-temperature compaction of nanopowder. A four-stage strategy (dissolution, diffusion, precipitation, and plastic deformation) is shown in Fig. 3c. Biomineralization is best suited to aqueous media and can reach the required density and morphology, as shown in Fig. 3d, e, but this is presently limited to thin films?
Water purification or the removal of the targeted chemicals from water is inspired by plant roots that allow selective water uptake and removal of selected nutrients from surrounding soil ${ }^{8}$.

There are many more processes that continually inspire us to develop artificial systems, such as energy storage inspired by biochemical energy storage ${ }^{9}$, protein production inspired by spider silk production ${ }^{10}$, and selfdegrading plastic inspired by natural decomposition ${ }^{11}$.

\section{Nature-inspired designs}

Nature has been splendidly designed to make life habitable. The nature-inspired design has therefore attracted great interest in recent times. Some of the examples in this series are shown in Fig. 4. Nature-inspired design can be adopted in two forms: surface design or structural design.

Surface design involves modification of a surface, such as in tailoring the wetting behavior. For example, hydrophobic plant leaves such as purple bauhinia (Phanera pupurea) and water cabbage (Pistia stratiotes) and hydrophilic nature rosy periwinkle (Cathranthus roseus $)^{12}$ show changed wettability when coated with a thin copper film. Hydrophobic leaves become highly absorbent, and hydrophilic leaves show low absorbance or high reflectance (due to the absence of nanostructuring over the leaf surface). The presence of nanostructures over the leaf surfaces significantly reduces reflectance, resulting in increased absorbance; this phenomenon inspired the use of nanostructured surfaces for wide wavelength (broadband) absorbance in solar absorber coatings ${ }^{12}$. Another surface patterning has been inspired by jay feathers, which show different colors at different incident angles.

Like the nature-inspired surface design, nature-inspired structural design can also offer new and enhanced properties (see Fig. 5). Toughness and strength are known as dichotomous trends, e.g., improved toughness reduces strength in metals. Similarly, in ceramics, the higher the compressive strength, the lower the toughness ${ }^{13}$. Imitating crystal structure architectures by three-dimensional (3D) printing in meso-microscale building blocks has replicated high damage-tolerant properties ${ }^{1}$.

\section{Nature-inspired materials}

This section is at the core of this article and discusses the concepts for developing materials meeting our needs and thus helping to achieve sustainability in our lifestyle. Nature-inspired materials are being developed with the intention of harnessing a certain type of functionality, which allows us to tap into a particular type of gain. A categorization of nature-inspired materials by virtue of the gain they provide is shown in Fig. 6, which shows that the type of gain could be (i) electrical, (ii) biological, (iii) chemical, (iv) mechanical, (v) sustainable, (vi) or a 


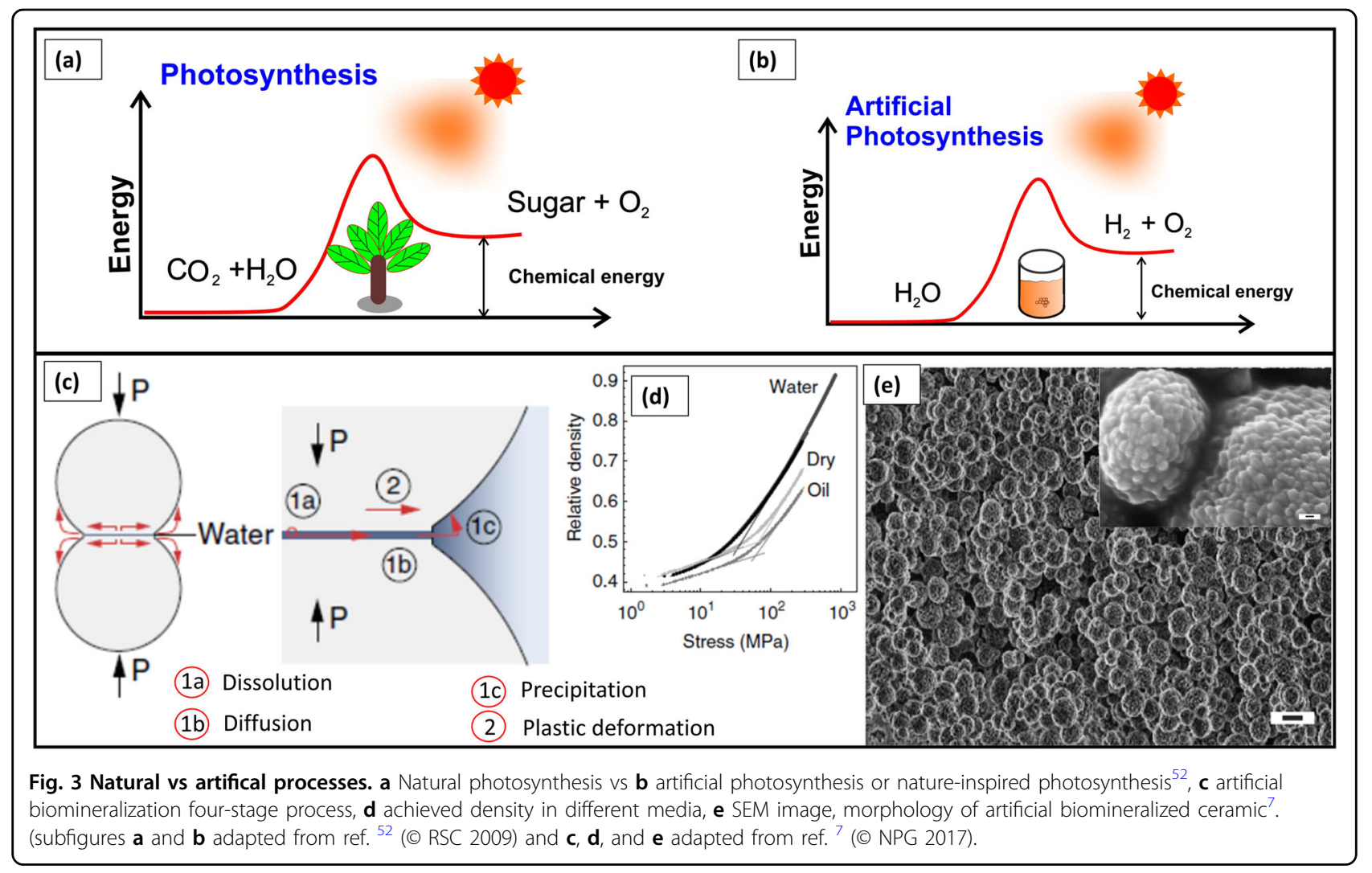

multiplicity of gains. The list of gains classified in Fig. 6 (and Table 1) is by no means exhaustive, but it enables suitably positioning new developments on this front. The individual categories shown in Fig. 6 are discussed further.

\section{Electrical gain}

A very interesting example in this category is that of an electric-eel mimicked miniature polyacrylamide hydrogel compartment that converts chemical energy to electrical energy ${ }^{14}$. This research can lead to the development of self-powered body implants. Another example is that of a nanomotor made of hyperbranched polyamide/L-arginine (HLA) ${ }^{15}$, which was mimicked from endogenous biochemical reactions in the human body. This development showed a pathway by which a nanomotor with no waste discharge can be created to facilitate many potential biological applications. Ravi et al. ${ }^{16}$ reported electrical charge storage in multiple layers of photoproteins isolated from Rhodobacter spheroids. The use of these proteins as charge storage media along with light harvesting may facilitate the development of a "self-charging biophotonic device". Teng et al. ${ }^{17}$ demonstrated the potential of bioinspired nervous signal transmission to simulate a neural ioncarried information system, as shown in Fig. 7.

\section{Biological gain}

This section describes examples of nature-inspired materials developed with an ambition to achieve biological gain. Recent research reported in this category includes examples of bionic 3D printed corals promoting space-efficient microalgal growth and they also possess outstanding photosynthetic quantum efficiencies ${ }^{18}$. This work is helpful in coral reef research and photobioreactor design. Another development inspired by observing the mechanism of plant seed dispersal units that can self-fold on differential swelling led to the fabrication of alumina compacts with bilayer architectures with control over shape change during sintering ${ }^{19}$. Biodegradable self-healing hydrogels for tissue repair were also reported ${ }^{20}$. In this work, the authors developed novel chitosan-cellulose nanofiber (CS-CNF) composite self-healing hydrogels with tunable self-healing properties. This research may lead to the development of a design rationale for hydrogels with better injectability and tissue regeneration potential. Gan et al. ${ }^{21}$ demonstrated a strategy for designing tough and adhesive hydrogels based on dynamic plant catechol chemistry, as shown below in Fig. 8.

\section{Chemical gain}

Research inspired by the hierarchical micro- and nanoscale features of diatoms has led to the fabrication 


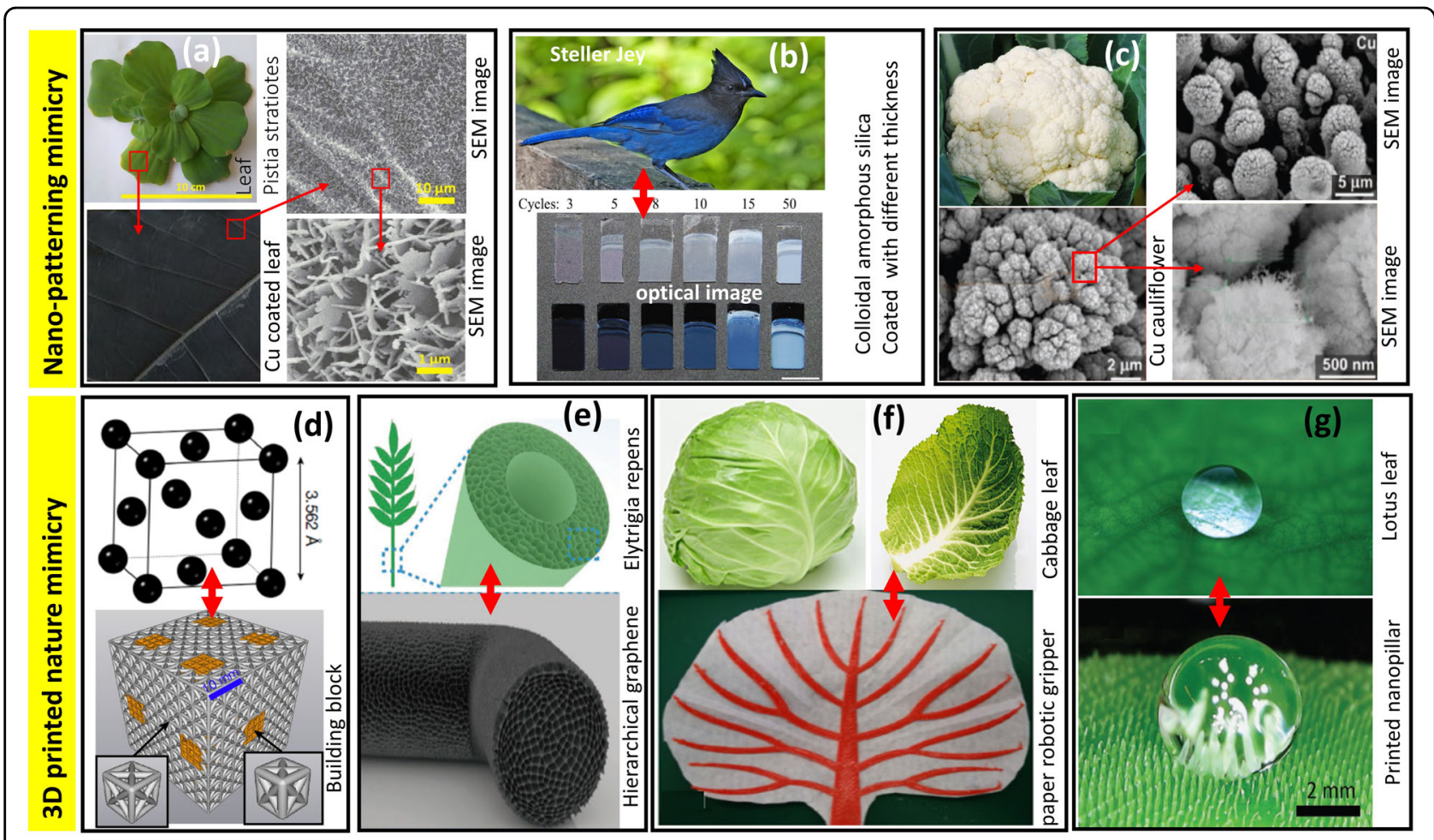

Fig. 4 Examples of nature-inspired bulk and surfaces. a Cu nanostructure for wide wavelength absorption generated through Phanera pupurea/ Pistia stratiotes leaf as template ${ }^{12} ; \mathbf{b}$ color alteration with different angles inspired by Steller's jay feather ${ }^{53} ; \mathbf{c}$ Cu nanostructure generated using laser, structure from cauliflower ${ }^{54} ; \mathbf{d}$ building block inspired by crystal structure ${ }^{1} ; \mathbf{e}$ hierarchical graphene ultralight inspired from Elytrigia repen ${ }^{51} ; \mathbf{f}$ soft robotic thermally driven paper gripper inspired by curling of cabbage leaf ${ }^{55} ; \mathbf{g} 3 \mathrm{D}$ printed nanopillar for superhydrophobic action inspired by a lotus

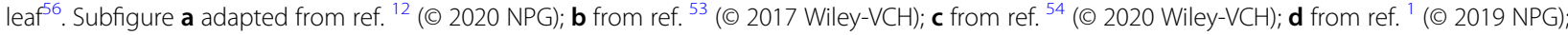
e from ref. ${ }^{51}$ (৫ 2019 Wiley VCH); f from ref. ${ }^{55}$ (৫ 2019 Springer) and g from rRef. ${ }^{56}$ (৫ 2019 Wiley VCH).

of a hierarchical diatomite membrane consisting of aligned microsized channels ${ }^{22}$. This diatomite membrane possesses both underwater superoleophobicity and superhydrophobicity and facilitates highly efficient oil/water separation. Another study ${ }^{23}$ reported on the development of amorphous calcium phosphate (ACP) doped with fluoride ions (FACP) to obtain materials with enhanced anticaries and demineralizing properties. This work made use of a biomineralization process and showed a pathway for preventative dentistry with the remineralization of dental hard tissues. This biomineralization strategy also helped to convert metal carbonate structures into lead halide perovskite semiconductors with tunable bandgaps, along with preservation of the 3D shape $\mathrm{e}^{24}$. This approach is promising, as calcium carbonate biominerals are converted into semiconductors, furnishing biological and programmable synthetic shapes. The development of carbonic anhydrase (CA)-based materials for the environmentally friendly sequestration of carbon dioxide $\left(\mathrm{CO}_{2}\right)$ under mild conditions can be helpful in arresting global warming $^{25}$. This research reported the development of CA-encapsulating silk protein hydrogels employing photoinduced dityrosine crosslinking followed by dehydration-mediated physical crosslinking. The current ambition for this research is to develop various progressive facade coatings employing biomimetic and bioinspired strategies ${ }^{26,27}$.

\section{Mechanical gain}

Nature-based materials can enhance the mechanical properties of materials, such as strength, toughness, hardness, and durability. Recently published research ${ }^{28}$ shows the development of a novel porous strut made of hollow cylindrical nanohydroxyapatite/polyamide, leading to faster osteointegration, and thus helping in cervical reconstruction. These struts possess the advantage of accelerated attachment/adhesion. Another example is that of the design of new adhesive devices inspired by insect footpads ${ }^{29}$. These footpads contain multiple hairs that secrete liquid, generating capillary force and thus helping the footpad stick to any surface. Taking the example of Drosophila, a type of fruit fly, the authors fabricated a new artificial adhesive device-a spatula-like fiber-framed adhesive device supported by nylon fibers with a gel material at the tip. 


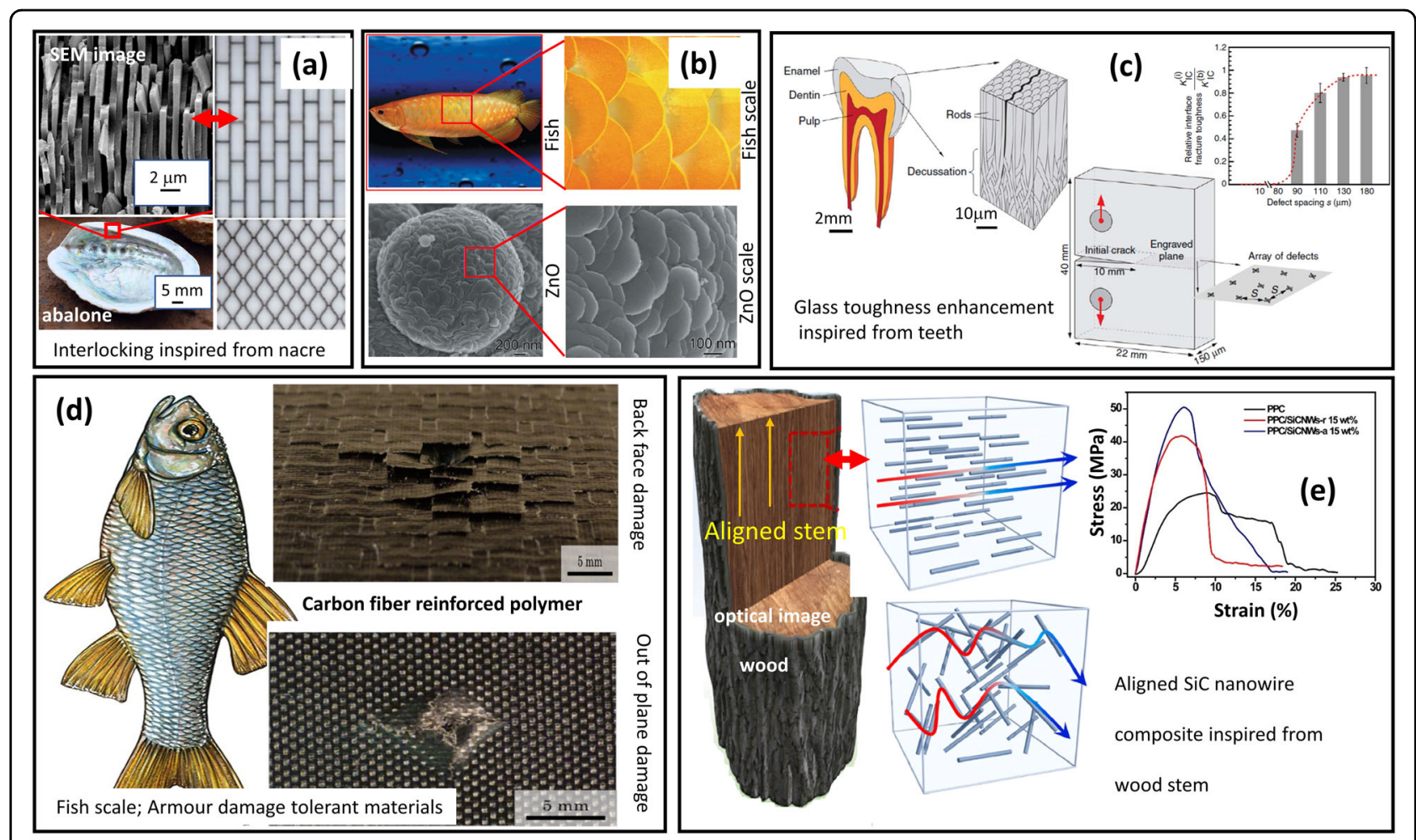

Fig. 5 Different nature-inspired examples. a Enhanced wall strength construction mimicry of interlocking aragonite plates in nacre ${ }^{57}$, b fish scale generated using $\mathrm{ZnO}^{58}$, c enhanced glass toughness inspired by tooth enamel ${ }^{59}$, d fiber-reinforced armor strength polymer inspired by fish scale ${ }^{60}$, and $\mathbf{e}$ the alignment of carbon nanotubes in nanocomposites inspired by wood stem ${ }^{61}$. Subfigure a adapted from ref. ${ }^{57}$ (๑) $2016 \mathrm{NPG);} \mathbf{b}$ from ref. ${ }^{58}$ (๑ 2015 NPG); c from ref. ${ }^{59}$ (৫ 2014 NPG); d from ref. ${ }^{60}$ (๑ 2020 Elsevier); e from ref. ${ }^{61}$ (๑ 2020 Elsevier).

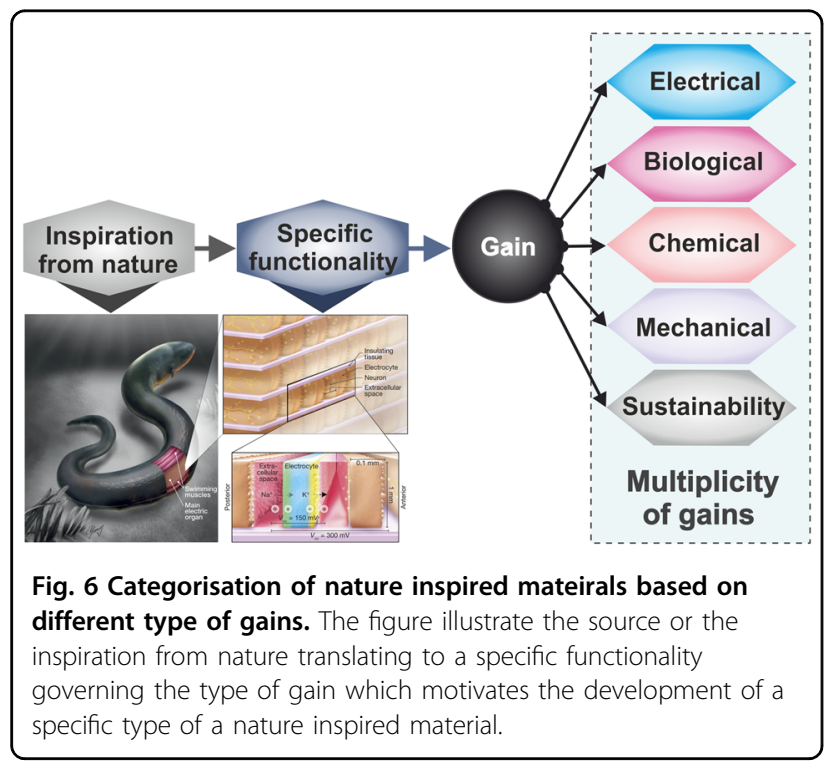

Libonati et al. ${ }^{30}$ reported a bone-inspired structure on fiber-reinforced composites. The geometry mimicked the osteonal secondary structure of mammalian bone. Bundles of unidirectional glass fibers (UDGF) were embedded into $\pm 45^{\circ}$ carbon fiber (CF) sleeves.
The orientation of the UDGF was orthogonal to the main osteon direction, providing a balance in the fiber orientation and ensuring good performance of the whole material in the transverse direction. The outer circumferential system was mimicked by a bidirectional woven GF fabric. The whole system was impregnated by epoxy resin. The design significantly boosted the fracture toughness when compared to a classic laminated composite. Chen et al. ${ }^{31}$ utilized PSeD-U elastomers with a unique physical and covalent hybrid crosslinking structure to mechanically and biologically develop skinlike materials. Other researchers ${ }^{32,33}$ reported that spider silk properties and architecture inspired the development of materials helping fog water harvesting and materials with enhanced mechanical properties. Chen et al. ${ }^{34}$ reported that Sarracenia trichome mimicked hierarchical microchannel organized material with superior fog water harvesting. Wang et al. ${ }^{35}$ designed and fabricated a two-dimensional (2D) spiderweb-like fog collector and a 3D cactus-like fog collector using direct laser structuring and origami techniques, as shown in Fig. 9.

Yang et al. ${ }^{36}$ developed neural probes or neuron-like electronics (NeuE) directed toward brain-machine 
Table 1 List of different nature-inspired materials classified according to the scheme shown in Fig. 6.

\begin{tabular}{|c|c|}
\hline Functionality & Application \\
\hline \multicolumn{2}{|c|}{ Nature-inspired materials triggered by achieving "Electrical gain" } \\
\hline $\begin{array}{l}\text { Electric-eel-inspired chemical to electrical energy } \\
\text { conversion }^{14}\end{array}$ & Artificial electrical organs \\
\hline Biological micromotors based on bacterium ${ }^{15}$ & Nature-inspired nanomotor \\
\hline Photosynthetic proteins ${ }^{16}$ & Self-charging biophotonic device \\
\hline \multicolumn{2}{|c|}{ Nature-inspired materials triggered by achieving "Biological gain" } \\
\hline Biological cooperativity of proteins ${ }^{64}$ & Protein fibrils \\
\hline Coral-algal symbiosis $^{18}$ & To grow microalgae with high spatial cell densities \\
\hline Tunable self-healing ${ }^{20}$ & Tissue repair \\
\hline Morphogenesis of biological structures ${ }^{65}$ & Biomorphs - Pneumatic shape-morphing structures \\
\hline Response of protein ${ }^{66}$ & Cancer biomarker detection \\
\hline $\begin{array}{l}\text { Plant seed dispersal units that self-fold on differential } \\
\text { swelling }{ }^{19}\end{array}$ & Self-shaping ceramic \\
\hline \multicolumn{2}{|c|}{ Nature-inspired materials triggered by achieving "Chemical gain" } \\
\hline Hierarchical micro- and nanoscale features of diatom ${ }^{22}$ & Diatomite membrane can selectively filter water in various oils \\
\hline Biomineralization $^{23}$ & Dental hard tissue \\
\hline Biomineralization ${ }^{24}$ & Perovskite cells \\
\hline Biomimicked dual crosslinking ${ }^{25}$ & Sequestration of carbon dioxide $\left(\mathrm{CO}_{2}\right)$ \\
\hline Hydrophobic paint (Lotusan) & Stocolor ${ }^{\oplus}$ Lotusan paint \\
\hline Self-healing & Airplane \\
\hline \multicolumn{2}{|c|}{ Nature-inspired materials triggered by achieving "Mechanical gain" } \\
\hline Fast osteointegration ${ }^{28}$ & Porous struts applied to (anterior cervical reconstruction of a goat) \\
\hline Functional properties of biological tissues ${ }^{31}$ & Tissues inspired bioelectronics \\
\hline Spider silk mimicked wettability ${ }^{32}$ & Biomimicked spindle-knot microfibers with cavity knots (named cavity-microfiber) \\
\hline $\begin{array}{l}\text { Subcellular structural features and mechanical properties } \\
\text { of neurons } s^{36}\end{array}$ & Neural probes or neuron-like electronics (NeuE) \\
\hline $\mathrm{Nacre}^{37}$ & Optical transparency and mechanically tough composites \\
\hline $\begin{array}{l}\text { Ultrafast water transport on the surface of a Sarracenia } \\
\text { trichome }^{34}\end{array}$ & Microfluidic \\
\hline Tooth enamel-mimicked columnar nanocomposites ${ }^{38}$ & Abiotic tooth enamel \\
\hline Mechanically response to external stimuli ${ }^{39}$ & Actuator, artificial muscles \\
\hline $\begin{array}{l}\text { Spider dragline silk based on the molecular assembly of } \\
\text { silk proteins }{ }^{33}\end{array}$ & Synthetic spider silks \\
\hline Fish scales and osteoderms ${ }^{67}$ & Bullet-proof protecto-flexible material \\
\hline
\end{tabular}

Recyclability ${ }^{68}$

Biodegradability (Shrilk)
Recyclable lightweight structures with hierarchical architectures, complex geometries, and unprecedented stiffness and toughness

Implantable foams, films, and scaffolds for surgical closures as well as regenerative medicine applications

Nature-inspired materials triggered by achieving "Multiplicity of gains"

Spider silk mimicked ${ }^{69}$

Humanoid robotics 
Table 1 continued

\begin{tabular}{ll}
\hline Functionality & Application \\
\hline Macroscopically ordered rod-like nanoapatites $^{70}$ & Aqueous liquid crystal, aqueous $\mathrm{Mg}(\mathrm{OH})_{2}$, and $\mathrm{Mg}_{3}\left(\mathrm{PO}_{4}\right)_{2} \mathrm{LCs}$ \\
Plant catechol chemistry & Tough and adhesive hydrogels \\
Adhesive nature of catechol chemistry & Switchable adhesive properties in a wet environment \\
Nature's Murray networks (Murray's law) $^{41}$ & Murray material \\
Biomimicked antireflective properties (insect compound $^{72}$ & Solar energy harvesting \\
eyes) $^{72}$ & \\
Artificial urushi (wetting) $^{73}$ & Coatings \\
Autonomous photomechanical actuation $^{74}$ & Flytrap \\
\hline
\end{tabular}

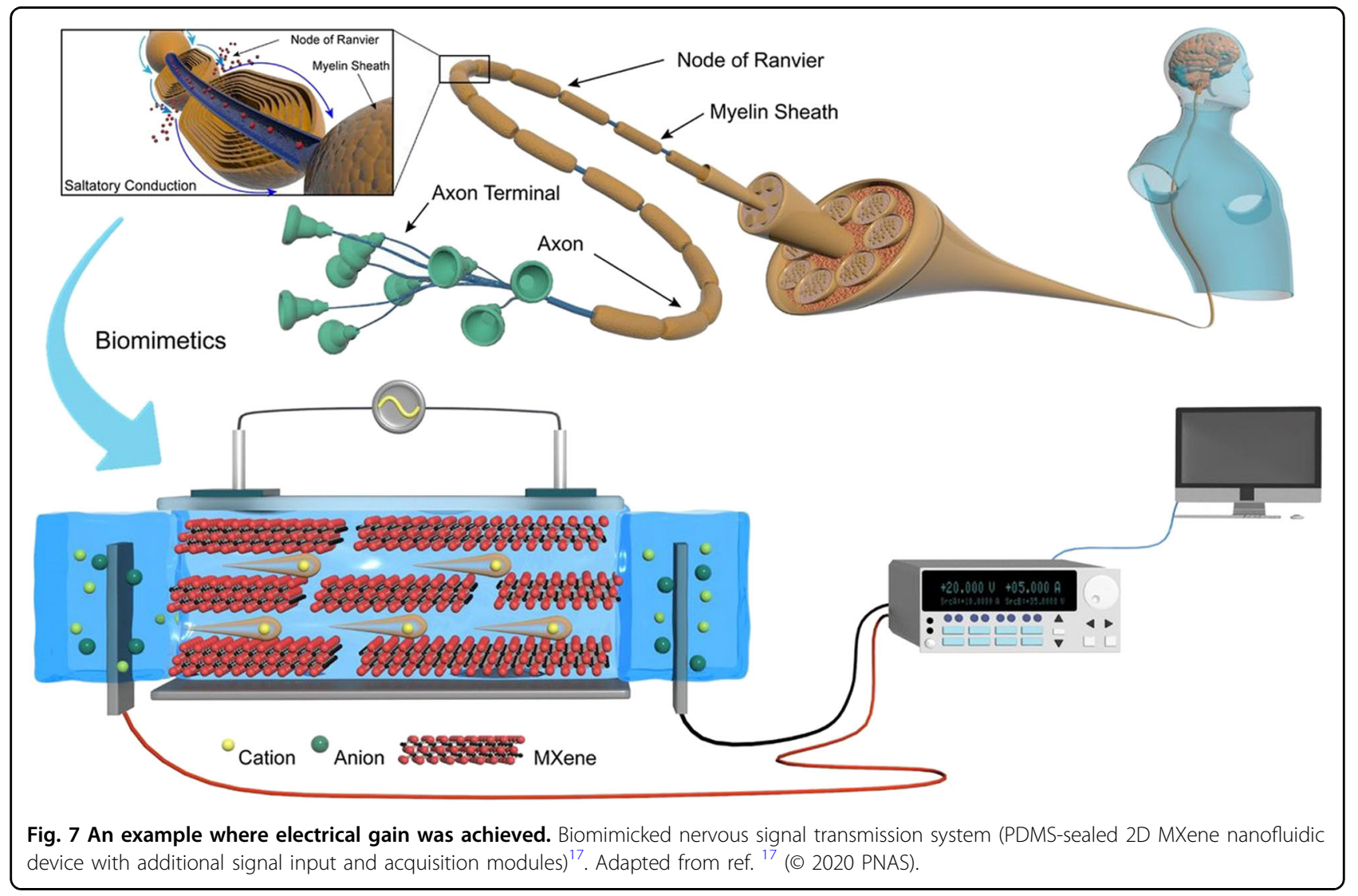

interfaces. Magrini et al. ${ }^{37}$ developed materials inspired by nacre-like architecture by combining antagonistic functional properties such as optical transparency and mechanical toughness. Yeom et al. ${ }^{38}$ reported enamelinspired columnar nanocomposites by the sequential growth of zinc oxide nanowire carpets followed by layer-by-layer deposition of a polymeric matrix with comparable mechanical properties. Deng et al. ${ }^{39}$ prepared hierarchically arranged helical fiber (HHF) actuators that can sense solvents/vapors and respond.

\section{Multiplicity of gains or collective gains}

The developments of materials described in this section are those that show multiple gains or a combination of several gains necessary to design a full system. An example of this is that of a human nerve or an optical eye for scotopic vision, as shown in Fig. 10.

Nerves are central systems that allow sensing in a human body, such as touch, perception, recognition, communication, and transmission. Developing bionic artificial nerves is vitally important for humanoids and 


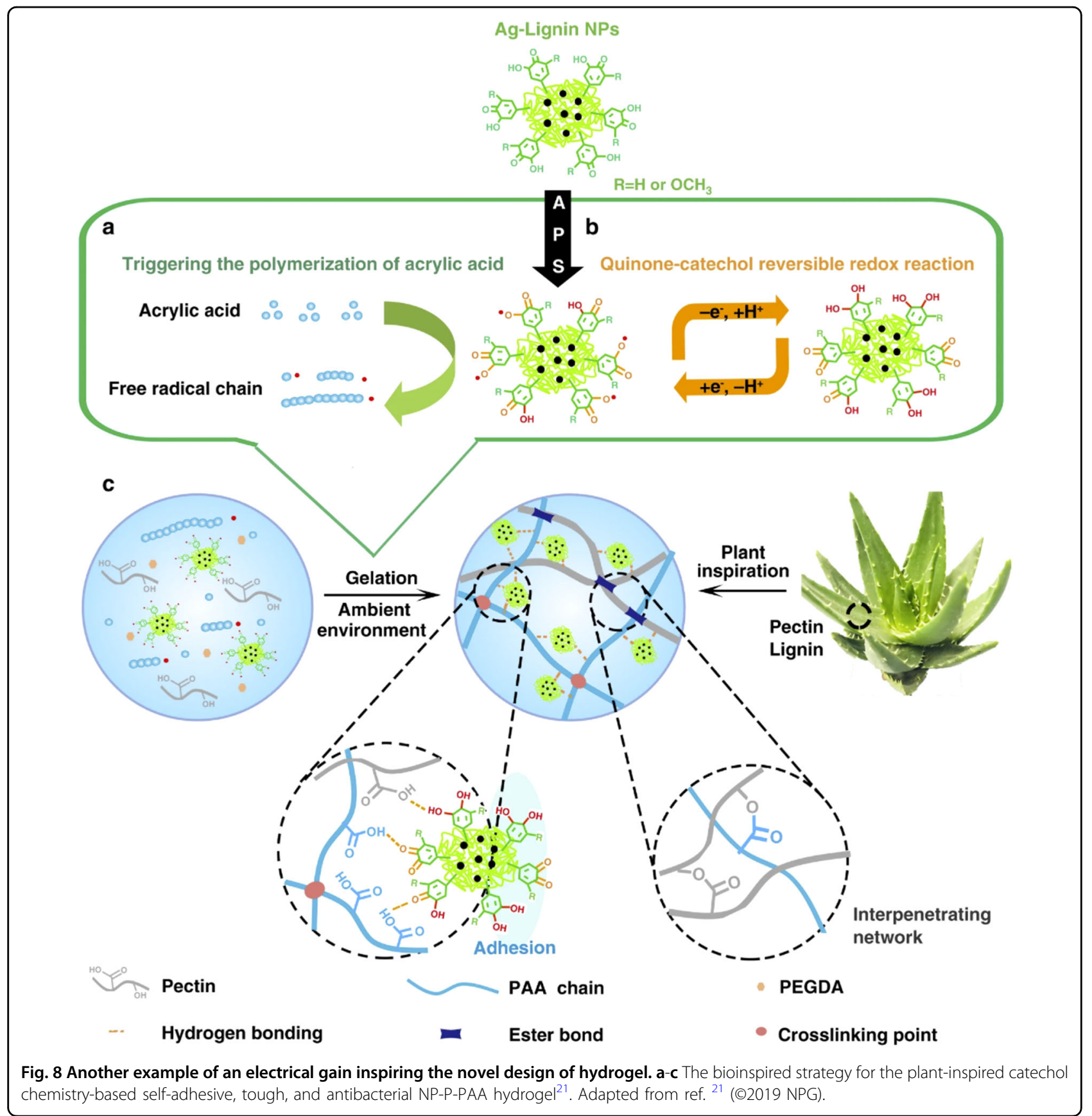

intelligent robots ${ }^{40}$. A recently developed artificial nerve is very efficient in transmitting mechanosensitive signals. It works based on an electrical double-layer structure, thus minimizing noise. It is envisaged that further developments will drive these artificial nerves to be able to sense temperature, humidity, and light to contribute to sophisticated neuroprosthetics. An electrotendon mechanically toughened by single-wall carbon nanotubes (SWCNTs) and electrically enhanced by PEDOT:PSS (poly(3,4-ethylenedioxythiophene) polystyrene sulfonate) can withstand more than 40,000 bending-stretching cycles without changes in conductivity. Various hierarchical designs in nature are guided by Murray's law, and utilizing this law, researchers developed materials whose pore sizes decrease across multiple scales and finally terminate in size-invariant units such as plant stems, leaf veins, and vascular and respiratory systems ${ }^{41}$. This approach ensures hierarchical branching and precise diameter ratios for connecting multiscale pores from macro to micro levels. It is envisaged that these Murray materials can enhance performance in photocatalysis, gas sensing, and Li-ion battery electrodes. 

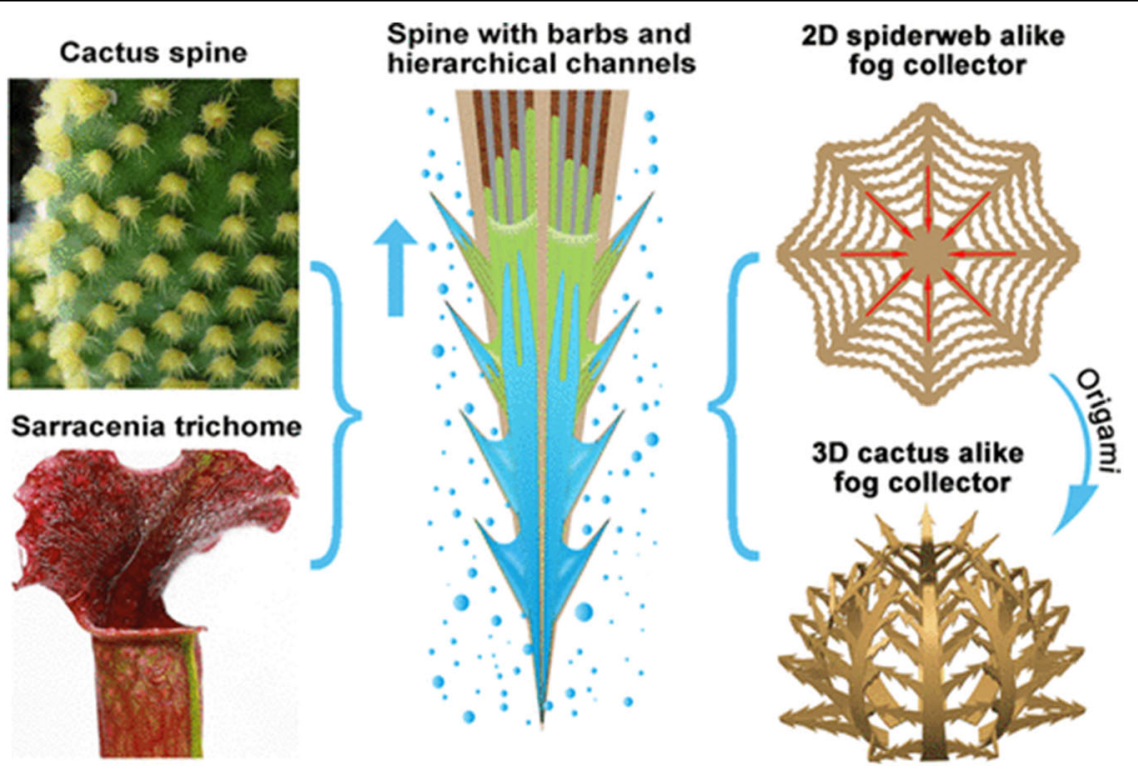

Fig. 9 Example of a mechanical gain. Figure shows a biomimicked spine with backward microbarbs and hierarchical microchannels for ultrafast water transport and efficient fog harvesting ${ }^{35}$. Adapted from ref. ${ }^{35}$ (๑2020 ACS).

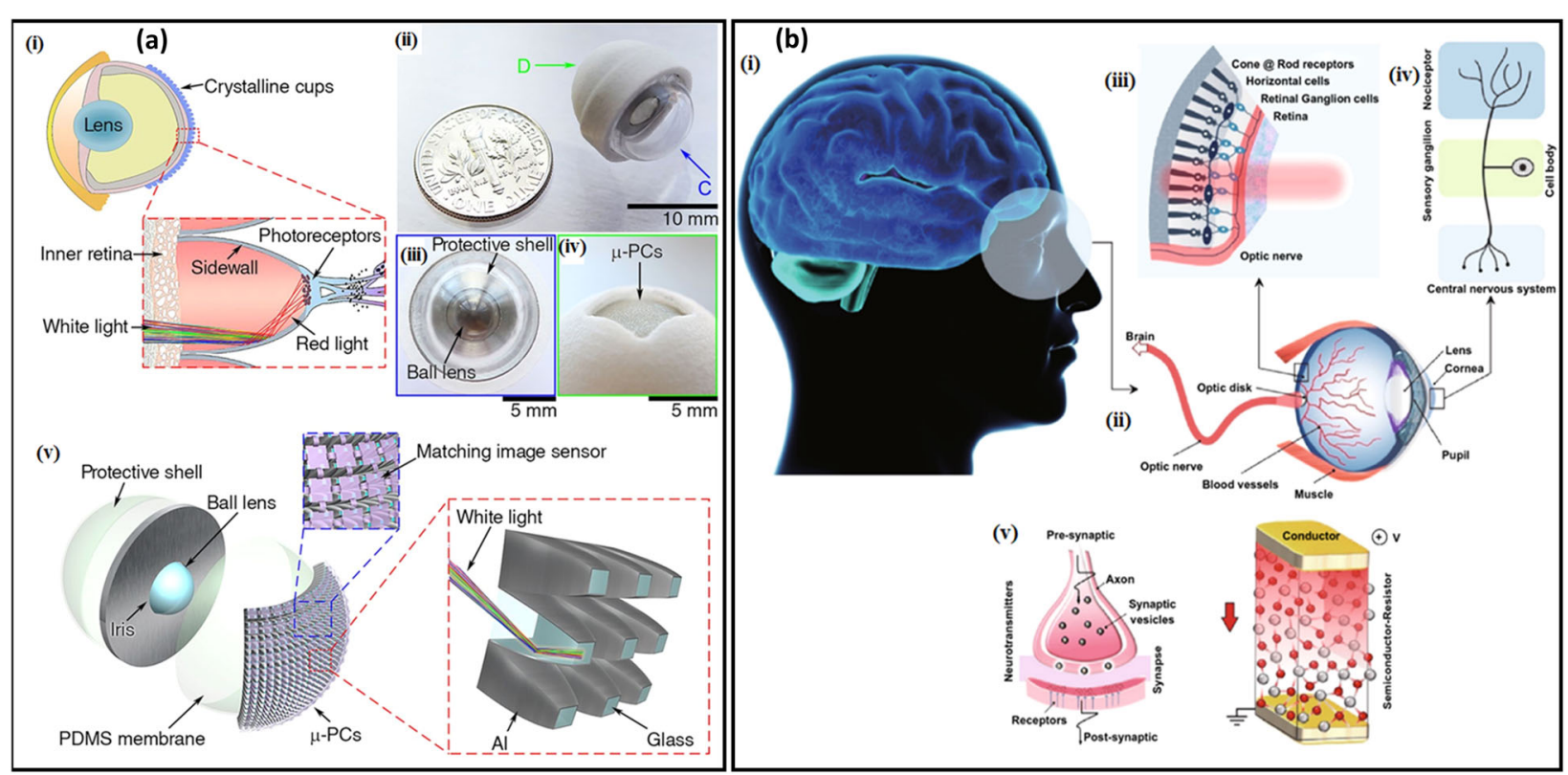

Fig. 10 Devices inspired from body parts. a Schematic illustrations and images of a natural eye of elephant nose fish and an artificial eye for scotopic vision ${ }^{62}$. b The scheme of human eye receptor and nociceptor system and its artificial counterparts in conductor/semiconductor/conductorsandwiched configuration ${ }^{63}$. Sub figure $\mathbf{a}$ adapted from ref. ${ }^{62}$ (@2016 PNAS); b adapted from ref. ${ }^{63}$ (@2020 Springer).

\section{Toward a holistic nature-inspired approach}

Conventional natural inspiration has been based on the direct copying/imitation of naturally occurring material structures with the expectation that these structures will meet the desired functionalities. However, with newly acquired knowledge, this concept is now achieving newer heights and horizons that can be referred to as fundamental design, where mimetics are now achieved only after the process is well understood in terms of its three main pillars: design, materials, and manufacturing. These are also the common pillars of an engineering design approach, which brings us to discuss a few fundamental 
Table 2 Nature-triggered vs engineering-triggered protocols.

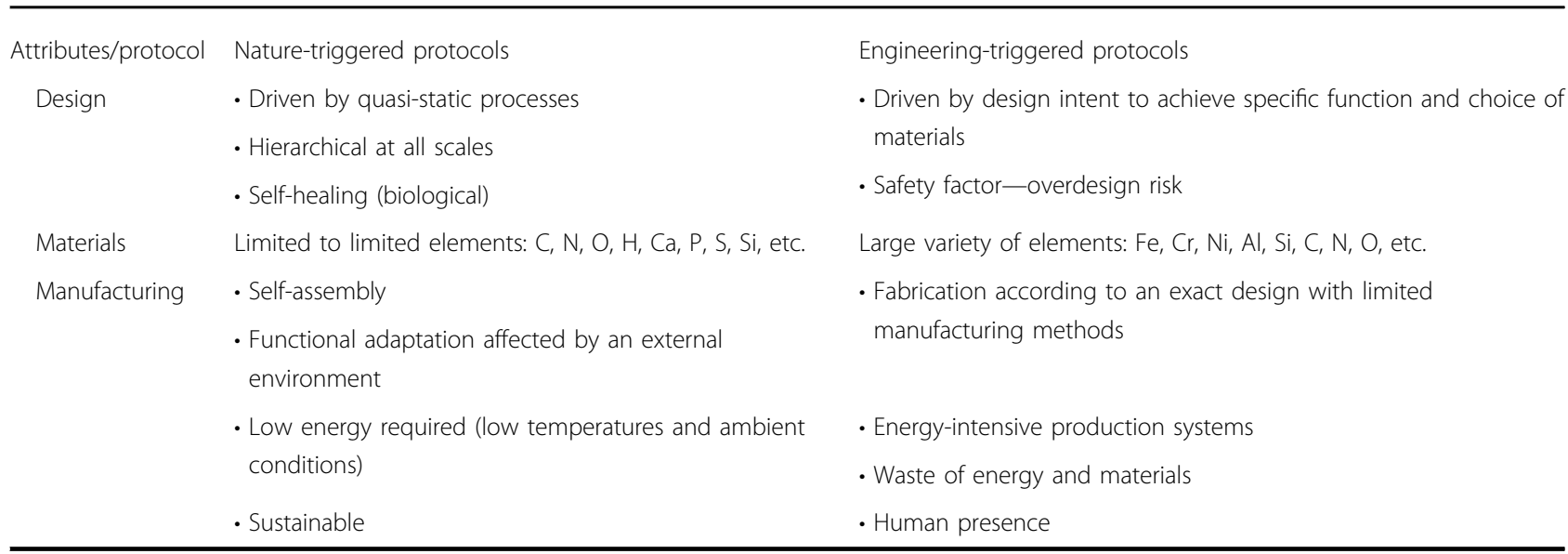

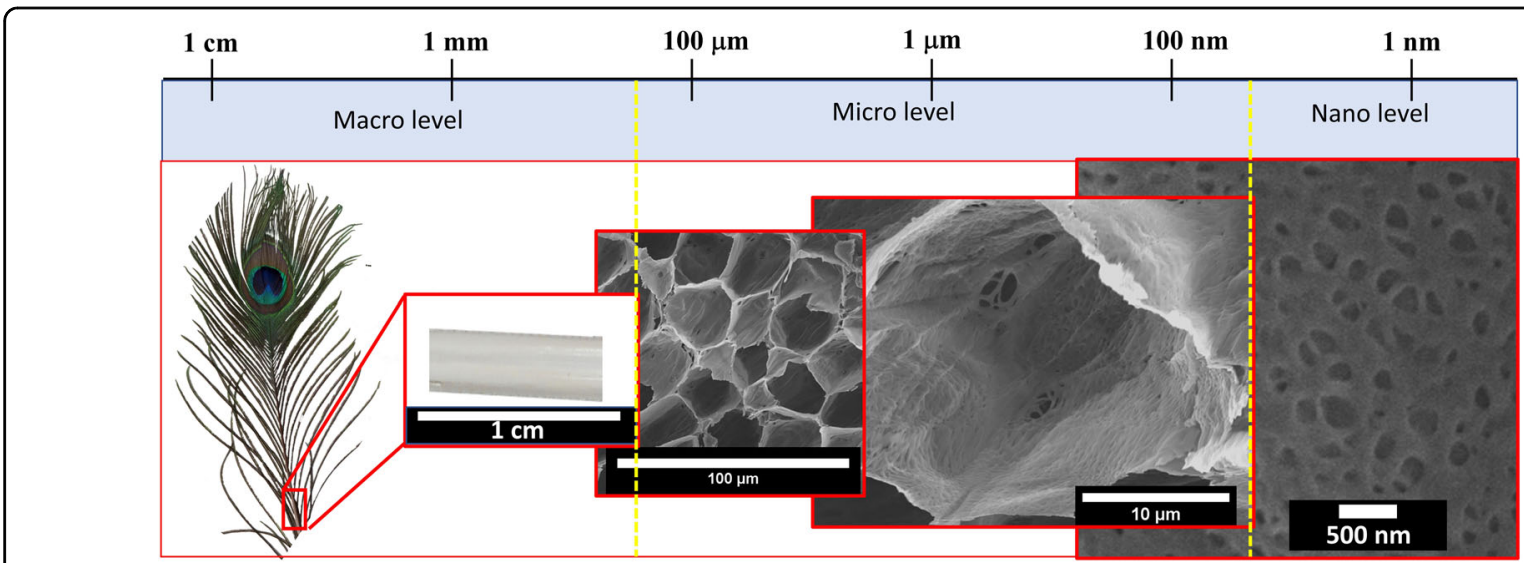

Fig. 11 Multilength scale design architecture of a peacock feather. Different size scale levels of intricate patterns enabled in naturally or biologically made materials (Peacock feathers and their rachis's SEM image).

differences between nature-triggered protocols and engineering-triggered protocols, which are shown in Table $2^{42}$.

In a nature-triggered protocol, the design is driven by a quasi-static thermodynamic process, which is a slowpaced process that allows the material system to maintain its internal equilibrium. These processes are self-run and do not need human intervention. Natural/biological materials possess different structural design elements (i.e., fibrous, helical, cellular, tubular elements, etc.) organized hierarchically due to the nature of the process driver. When comparing natural and synthetic materials in terms of their properties, such as strength and toughness, one might consider that synthetic materials have superior performance. However, nature assembles these relatively weak constituents into hierarchical composite structures that exhibit impressive combinations of strength and toughness. Thus, natural materials owe their superiority to the design of their structural hierarchical synergistic material systems, whereas engineering or synthetic materials rely on their inherent properties to guide the design.

Additionally, the production of natural materials is a slow bottom-up approach in which materials grow, selfassemble, and adapt to the ambient environment rather than being specifically designed and restricted as engineered materials. This bottom-up approach allows natural materials to be hierarchical at all scales. For example, the peacock feather rachis design was unveiled using scanning electron microscopy (SEM), as shown in Fig. 11. The intricate way by which mother nature enweaves the features from the nano to the macro level to make its creations robust and lightweight is an art form, as is the capability of nature to design biomaterials with multifunctionality, such as self-healing properties (biological materials) stemming from environment adaptation. 
As natural materials usually grow under ambient conditions, they do not require high energy for fabrication, similar to engineering materials. In addition, the sustainability of nature-triggered protocols is far superior to that of engineering-triggered protocols. Due to these differences between nature-triggered protocols and engineeringtriggered protocols, a better approach to nature inspiration would be adaptation and not blind imitation.

The drivers behind the pursuits of nature inspiration (encompassing bioinspiration) influence the strategy applied. As such, one can identify functional problemsolving through either problem-driven nature inspiration or solution-driven nature inspiration. These two approaches differ in the initial steps, as these are the steps where inspiration and ideas play a large role in design but converge to the same outcome. After the identification of biological models, the process becomes systematic.

The biomimicry design spiral ${ }^{43}$ was the steppingstone on which other design approaches such as the DTU biocard $^{44}$, the Biomimicry 3.8 DesignLens ${ }^{45}$, the ISO18458:2015 standard, the unified problem-driven process of biomimetics ${ }^{46}$, and the solution-driven process $^{47}$ were developed. A design spiral inspired by the biomimicry design spiral is shown in Fig. 12.

The individual steps or language may differ between the problem-driven processes, yet they all follow a common trend that was congregated in the unified problem-driven process of biomimetics introduced by Fayemi et al. $(2017)^{46}$. While this approach was developed for biomimetic design, its strategies likewise apply to natureinspired design with a change in terminology to encompass living and nonliving entities: biology to nature. The nature-expanded unified problem-driven approach can then be expressed in eight main steps as follows:

1. Problem analysis: Assess the situation in the case where no problem has been pinpointed yet or describe the problem previously identified.

2. Abstract technical problem: Identify context and constraints to define the function required.

3. Transpose to nature: Formulate the function required into a question toward nature and investigate how nature can achieve that goal. Careful question formulation is required, as the results are highly sensitive to the formulation.

4. Identify potential natural models: Through a literature search, natural models (including biological models) can be identified.The accumulated knowledge obtained at this step on both the technological and natural levels might necessitate revisiting the first three steps, thus forming an iterative loop.

5. Selecting a natural model of interest: Select a natural model from the identified models.

6. Abstract natural strategy: Understand the workings of the selected natural models and detach them from the

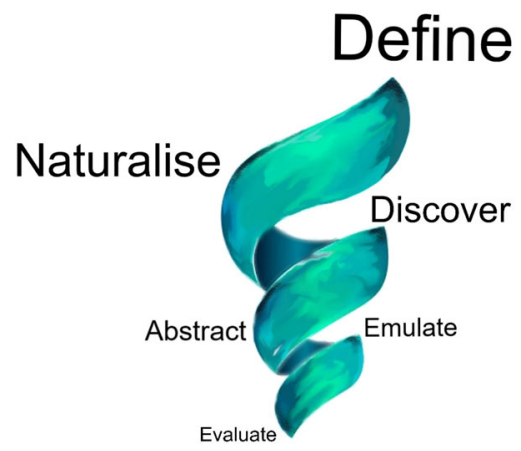

Fig. 12 The concept of design spiral. The figure shows the concept of a spiral adopted from the Biomimicry research institute.

natural entity. As a direct transition from nature to technology is impractical in most cases, the combination of several natural strategies is vital to solve the initial problem through a transferrable functional model.

7. Transpose to technology: To express the natural solution in technical terms, technological knowledge is crucial to allow implementation in the technical world.

8. Implementation and testing: Effective conversion of natural strategies to technology and subsequent implementation and testing will result in a successful conclusion of the cycle and the introduction of a nature-inspired design. In the case of unsatisfactory results, the process is repeated within either phase 1 (steps 1 to 3 ) or 2 (steps 4 to 8 ).

An illustration of the workings of the unified problembased approach applied to the development of a dynamic thermoregulatory material inspired by squid $\operatorname{skin}^{48}$ is shown in Fig. 13.

For the solution-driven process, it has been explained ${ }^{47}$ that this approach stands out from the problem-driven approach in natural solution identification as being the step that initiates the design process. This is done through observation, as this is the stage where inspiration and curiosity play a role. Numerous examples can be expressed, some that are obvious and that everyone has encountered, like observing droplets forming on numerous plant leaves and bird feathers such as pigeons, the ability of a chameleon to change color to regulate its temperature and communicate, and many others.

Since nature-inspired design is a multidisciplinary process, the challenge lies in the identification of the natural function to identify specific applications, as it is difficult to transfer natural concepts and terminologies into an engineering perspective in the exact same way. This has led to slow progress in the field. Therefore, several attempts have been made to facilitate the translation of concepts and terminologies between nature and 


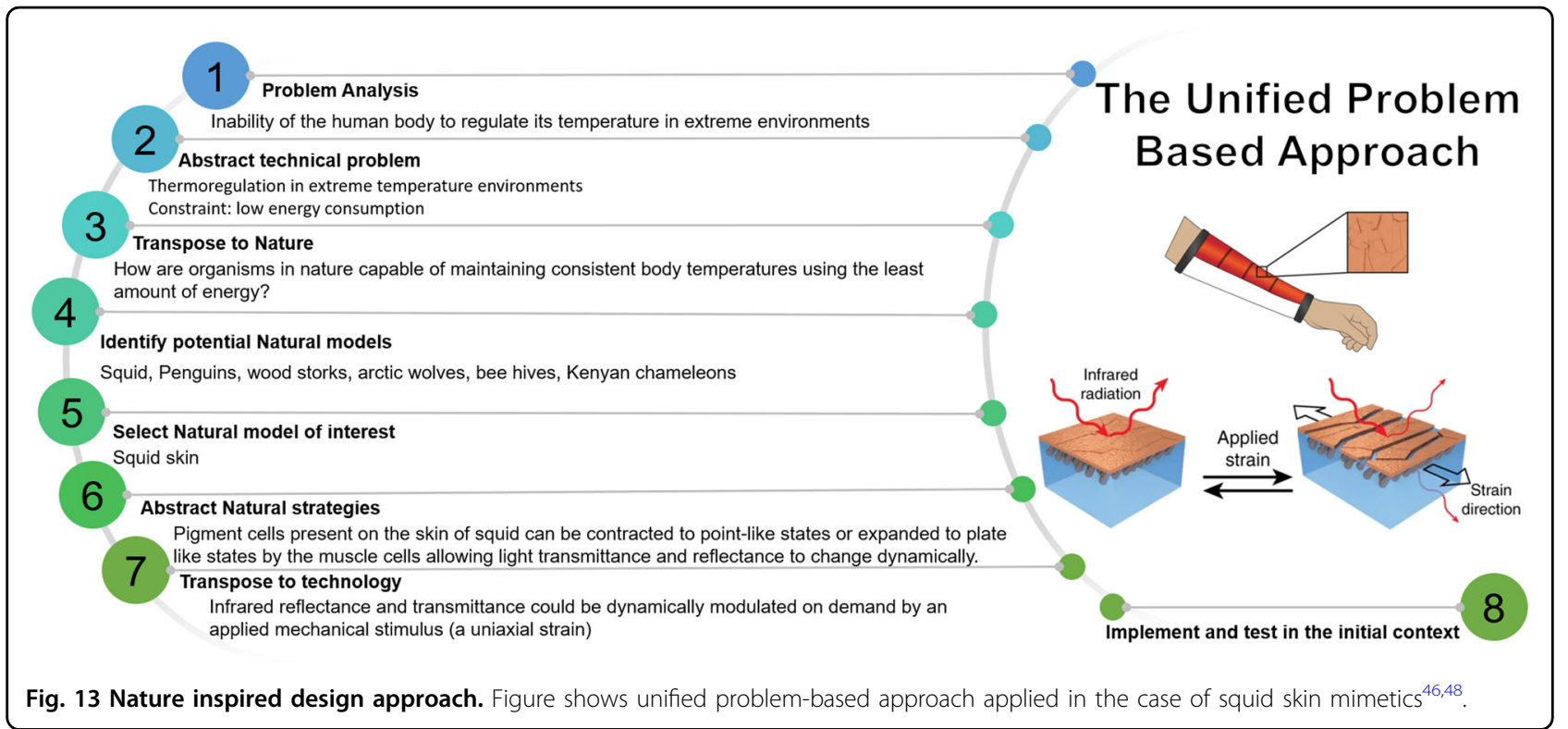

engineering and aid engineers in finding the functions and solutions that are suitable for their application. Intuitively, efforts have been made toward the creation of databases to gather hundreds of observed natural phenomena and classify them according to their function, such as AskNature and the bionics system database. Using such platforms and established knowledge, superior natureinspired materials can be designed.

However, as discussed previously, there is a difference between natural fabrication modes and engineering manufacturing, which limits the flexibility of the design. However, nature inspiration not only applies to the structure and function but is also used in process development. For instance, biomineralization is one of the processes inspired by nature. Nevertheless, due to the limitations still existing in fabrication modes, most biomineralization attempts have underachieved compared to their natural counterparts, as they are slow and can only be used to produce small prototypes exhibiting inferior mechanical properties ${ }^{49}$. With new advances in fabrication methods, designs can now be made to be more flexible, and manufacturing can be agile and smarter with low waste, thus contributing to sustainability, one of the resolves of nature mimicry.

\section{Author's views and further prospects}

Nature manifests its construction using the tiniest form of matter by taking a minimum energy approach akin to self-assembly type of processes. During the last two decades, nanoscience/nanotechnology has helped to improve the current understanding of the nanoscale world, which is the length scale at which nature begins its construction, although the time scale is too large. Nature can easily create multiple gain components, e.g., a human finger that can perceive pressure, hotness/coolness, can feel the wind flowing, inform about any damage (pain), can move on the instruction of the brain, enabled with self-healing ability on any cut, can grip things, and leave behind a footprint (fingerprints). The engineering world has yet to mature enough to manifest such complex multiple gains so swiftly and readily. Further advances are required in materials, design, manufacturing, and sensing to unlock nature's puzzle.

A biosystem is associated with three aspects: (i) miniaturization (many functions introduced in a small volume), (ii) organic-inorganic hybridization (introducing strength, durability, flexibility, etc.), and (iii) hierarchy (network construction from nano- to millimeter work function).

Currently, nature-inspired materials, processes, and designs still lack a hierarchical network, which requires a better control system to be developed. An even larger challenge is the practical realization of nature-inspired materials at a commercial scale, which comes down to their scalable and affordable production. Therefore, material developments must resonate with manufacturing developments.

Manufacturing techniques can be classified into three main categories: (i) those based on the removal of material, herein referred to as subtractive methods, (ii) those involving addition of material (deposition), herein referred to as additive methods, and (iii) techniques involving no addition or removal of material. Based on this categorization, some prime candidate technologies and newly emerging techniques currently being used for the fabrication of nature-inspired materials are shown in Fig. 14.

Among other techniques, 3D printing is gaining much attention due to the flexibility of the shapes it offers. Future potentials of 3D printing have prospects of copying 


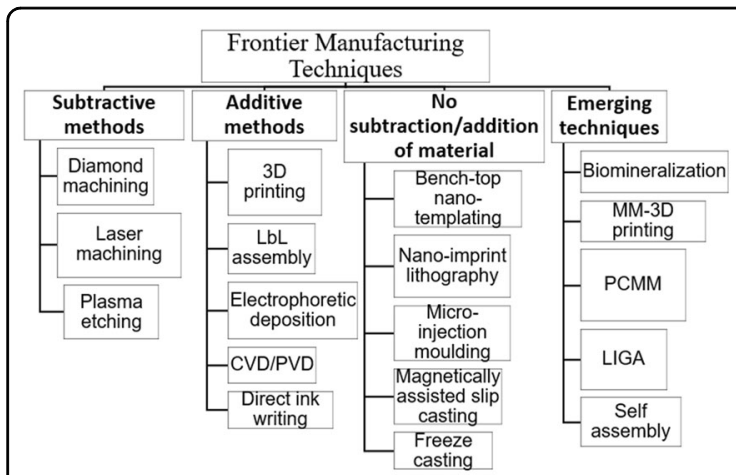

LbL: Layer by layer; CVD: Chemical vapour deposition; PVD: Physical vapour deposition; MM3D: Multimaterial multinozzle 3-dimensional printing; PCMM: Photochemical micromachining; LIGA: German acronym for Lithographie, Galvanoformung, Abformung

Fig. 14 Various manufacturing techinques developed and used so far to fabricate nature inspired materials. Figure shows various conventional and manufacturing techniques used to obtain nature mimetics.

natural architecture such as biomimetic scaffolds inspired from spinal $\operatorname{cords}^{50}$, an ultralight biomimetic hierarchical structure inspired by cellular structure ${ }^{51}$, damage-tolerant building blocks inspired by crystal structures ${ }^{1}$, etc. With the emergence of multimaterial multinozzle 3D printing (MM3D), one can extend the bandwidth or the range of materials that can be fabricated in a scalable way at once with significantly high precision.

There are challenges associated with the design of nature-inspired materials; for example, the smallest feature size for most production scale machines is in the range of hundreds of microns. Nonetheless, in powder bed processes, the trapped powder needs to be removed so the smallest size of voids is limited. After taking process constraints into account, initial designs are established, and parametric modeling is conducted. Consequently, simulations can be performed to optimize the design according to the function. However, the timescale over which any simulation is performed cannot match the experimental scale range of a few femtoseconds to a few weeks or years. In some cases, where the structure-function relationship is not fully understood, hands-on sampling and testing with a systematic design of experiments (DOE) approach can be employed. Intuitively, after optimization either through simulations or through sampling, prototyping and testing is required, which requires multidisciplinary efforts. To an extent, the proliferation of Artificial intelligence and machine learning has now started to emerge as a newer effort in this direction.

These are the most pressing challenges in imitating intricate hierarchical patterns and producing nature-inspired materials. As such, natural materials have the capability of self-decomposition and this process does not create an adverse ecological impact. However, artificial materials do not possess the same recyclability, and the pollution caused by plastics is a prime example of human intervention in Nature's ecosystem. On a triple bottom line (environment, economic, and social aspects), artificial materials are yet to fully adopt green manufacturing routes. Work on considering the sustainability of functional nature-inspired materials is a grand engineering challenge.

In view of these challenges, the following future directions are noteworthy:

1. Precision-at-scale production of nature-inspired materials

2. Achieving the lifetime and recyclability of natureinspired materials akin to nature

3. Making nature-inspired materials/design a fully digitalized process to be guided by predictive modeling and simulations

4. Identifying new sectors where these developmental materials can be deployed to reduce carbon footprint to address sustainable development goals (SDGs).

\section{Acknowledgements}

All authors greatly acknowledge the financial support provided by the UKRI via Grants No. EP/L016567/1, EP/S013652/1, EP/S036180/1, EP/T001100/1, and EP/ T024607/1, the Royal Academy of Engineering via Grants No. IAPP18-19 295, TSP1332, and EXPP2021 1\277, EURAMET EMPIR A185 (2018), the EU Cost Action (CA15102, CA18125, CA18224, and CA16235), and the VC Fellowship from Cranfield University and the Newton Fellowship award from the Royal Society (NIF\R1\191571). We also acknowledge financial support from the European Regional Development Funds (ERDF) sponsored A2i project as well as the feasibility study awarded by the EPSRC TFIN+ (EP/N026402/1) to LSBU. Wherever applicable, the work made use of Isambard Bristol, UK supercomputing service accessed by a Resource Allocation Panel (RAP) grant as well as ARCHER resources (Project e648).

\section{Author details}

${ }^{1}$ School of Engineering, London South Bank University, London SE10 AA, UK. ${ }^{2}$ School of Aerospace, Transport and Manufacturing, Cranfield University, Bedford MK43 OAL, UK. ${ }^{3}$ Department of Mechanical Engineering, Shiv Nadar University, Gautam Budh Nagar 201314, India

\section{Data availability}

As this is a review paper, no new research data was generated in this work.

\section{Conflict of interest}

The authors declare no competing interests.

\section{Publisher's note}

Springer Nature remains neutral with regard to jurisdictional claims in published maps and institutional affiliations.

Received: 30 November 2020 Revised: 27 May 2021 Accepted: 4 June 2021. Published online: 30 July 2021

\section{References}

1. Pham, M.-S., Liu, C., Todd, I. \& Lertthanasarn, J. Damage-tolerant architected materials inspired by crystal microstructure. Nature 565, 305-311 (2019).

2. Darmanin, T. \& Guittard, F. Superhydrophobic and superoleophobic properties in nature. Mater. Today 18, 273-285 (2015). 
3. Gust, D., Moore, T. A. \& Moore, A. L. Solar fuels via artificial photosynthesis. Acc. Chem. Res. 42, 1890-1898 (2009).

4. Wang, J., Zhu, T. \& Ho, G. W. Nature-inspired design of artificial solar-to-fuel conversion systems based on copper phosphate microflowers. ChemsusChem. 9, 1575-1578 (2016).

5. Xi, B. et al. TiO2 thin films prepared via adsorptive self-assembly for selfcleaning applications. ACS Appl Mater. Interfaces 4(2), 1093-1102 (2012).

6. Yao, S. et al. Biomineralization: from material tactics to biological strategy. Adv. Mater. 29, 1605903 (2017).

7. Bouville, F. \& Studart, A. R. Geologically-inspired strong bulk ceramics made with water at room temperature. Nat. Commun. 8, 14655 (2017).

8. Freeman, E. C., Soncini, R. M. \& Weiland, L. M. Biologically inspired water purification through selective transport. Smart Mater. Struct. 22, 014013 (2012).

9. Wang, H., Yang, Y. \& Guo, L. Nature-inspired electrochemical energy-storage materials and devices. Adv. Energy Mater. 7, 1601709 (2017).

10. Kronqvist, $\mathrm{N}$. et al. Efficient protein production inspired by how spiders make silk. Nat. Commun. 8, 15504 (2017).

11. Guan, Q.-F., Yang, H.-B., Han, Z.-M., Ling, Z.-C. \& Yu, S.H. An all-natural bioinspired structural material for plastic replacement. Nat. Commun. 11, 5401 (2020).

12. Dao, T. D. et al. Bio-inspired broadband absorbers induced by copper nanostructures on natural leaves. Sci. Rep. 10, 3243 (2020).

13. Launey, M. E. \& Ritchie, R. O. On the fracture toughness of advanced materials. Adv. Mater. 21, 2103-2110 (2009).

14. Schroeder, T. B. H. et al. An electric-eel-inspired soft power source from stacked hydrogels. Nature 552, 214-218 (2017).

15. Wan, M. et al. Bio-inspired nitric-oxide-driven nanomotor. Nat. Commun. 10, 966 (2019).

16. Ravi, S. K. et al. Photosynthetic apparatus of Rhodobacter sphaeroides exhibits prolonged charge storage. Nat. Commun. 10, 902 (2019).

17. Teng, Y. et al. Bioinspired nervous signal transmission system based on twodimensional laminar nanofluidics: from electronics to ionics. Proc. Natl Acad. Sci. USA 117, 16743 (2020).

18. Wangpraseurt, D. et al. Bionic 3D printed corals. Nat. Commun. 11, 1748 (2020).

19. Bargardi, F. L., Le Ferrand, H., Libanori, R. \& Studart, A. R. Bio-inspired selfshaping ceramics. Nat. Commun. 7, 13912 (2016).

20. Cheng, K.-C., Huang, C.-F., Wei, Y. \& Hsu, S.-H. Novel chitosan-cellulose nanofiber self-healing hydrogels to correlate self-healing properties of hydrogels with neural regeneration effects. NPG Asia Mater. 11, 25 (2019).

21. Gan, D. et al. Plant-inspired adhesive and tough hydrogel based on Ag-Lignin nanoparticles-triggered dynamic redox catechol chemistry. Nat. Commun. 10 1487 (2019)

22. Lo, Y.-H., Yang, C.-Y., Chang, H.-K., Hung, W.-C. \& Chen, P.-Y. Bioinspired diatomite membrane with selective superwettability for oil/water separation. Sci. Rep. 7, 1426 (2017).

23. lafisco, M. et al. Fluoride-doped amorphous calcium phosphate nanoparticles as a promising biomimetic material for dental remineralization. Sci. Rep. 8, 17016 (2018)

24. Holtus, T. et al. Shape-preserving transformation of carbonate minerals into lead halide perovskite semiconductors based on ion exchange/insertion reactions. Nat. Chem. 10, 740-745 (2018).

25. Kim, C. S., Yang, Y. J., Bahn, S. Y. \& Cha, H. J. A bioinspired dual-crosslinked tough silk protein hydrogel as a protective biocatalytic matrix for carbon sequestration. NPG Asia Mater. 9, e391-e391 (2017).

26. https://www.sto.com/en/portfolio/facades/intelligent-technologies/standard. html. Accessed on 27 May 2021.

27. Wu, Z. L. \& Gong, J. P. Hydrogels with self-assembling ordered structures and their functions. NPG Asia Mater. 3, 57-64 (2011).

28. Liang, $X$. et al. In vivo evaluation of porous nanohydroxyapatite/polyamide 66 struts in a goat cervical fusion model. Sci. Rep. 10, 10495 (2020).

29. Kimura, K.-I., Minami, R., Yamahama, Y., Hariyama, T. \& Hosoda, N. Framework with cytoskeletal actin filaments forming insect footpad hairs inspires biomimetic adhesive device design. Commun. Biol. 3, 272 (2020).

30. Libonati, F. et al. Bone-inspired enhanced fracture toughness of de novo fiber reinforced composites. Sci. Rep. 9, 3142 (2019).

31. Chen, S. et al. Mechanically and biologically skin-like elastomers for biointegrated electronics. Nat. Commun. 11, 1107 (2020).

32. Tian, Y. et al. Large-scale water collection of bioinspired cavity-microfibers. Nat. Commun. 8, 1080 (2017)

33. Peng, Q. et al. Recombinant spider silk from aqueous solutions via a bioinspired microfluidic chip. Sci. Rep. 6, 36473 (2016).
34. Chen, $\mathrm{H}$. et al. Ultrafast water harvesting and transport in hierarchical microchannels. Nat. Mater. 17, 935-942 (2018).

35. Wang, J. et al. Laser direct structuring of bioinspired spine with backward microbarbs and hierarchical microchannels for ultrafast water transport and efficient fog havesting. ACS Appl Mater. Interfaces 12, 21080-21087 (2020).

36. Yang, $X$. et al. Bioinspired neuron-like electronics. Nat. Mater. 18, 510-517 (2019).

37. Magrini, T. et al. Transparent and tough bulk composites inspired by nacre. Nat. Commun. 10, 2794 (2019).

38. Yeom, B. et al. Abiotic tooth enamel. Nature 543, 95-98 (2017).

39. Deng, J. et al. Preparation of biomimetic hierarchically helical fiber actuators from carbon nanotubes. Nat. Protoc. 12, 1349-1358 (2017).

40. Liao, X. et al. A bioinspired analogous nerve towards artificial intelligence. Nat. Commun. 11, 268 (2020)

41. Zheng, X. et al. Bio-inspired Murray materials for mass transfer and activity. Nat Commun. 8, 14921 (2017).

42. Hunter, P. From imitation to inspiration: biomimicry experiences a revival driven by a more systematic approach to explore nature's inventions for human use. EMBO Rep. 18, 363-366 (2017).

43. https://biomimicry.org/biomimicry-design-spiral/. Accessed on 27 May 2021.

44. Anker, L. T. Do biomimetic students think outside the box? In Proc. of the International Conference on Engineering Design (eds Maier, A. et al.) 543-551 (ICED, 2017).

45. Alessandro, B., Caterina, C., Marinella, L., Francesco, R. \& Alessandro, Z. Biomimicry thinking: methodological improvements and practical implementation. Bioinspired Biomim. Nanobiomaterials 6, 87-101 (2017).

46. Fayemi, P. E., Wanieck, K., Zollfrank, C., Maranzana, N. \& Aoussat, A. Biomimetics: process, tools and practice. Bioinspir. Biomim. 12, 011002 (2017).

47. Helms, M., Vattam, S. S. \& Goel, A. K. Biologically inspired design: process and products. Des. Stud. 30, 606-622 (2009).

48. Leung, E. M. et al. A dynamic thermoregulatory material inspired by squid skin. Nat. Commun. 10, 1947 (2019).

49. Wegst, U. G. K., Bai, H., Saiz, E., Tomsia, A. P. \& Ritchie, R. O. Bioinspired structural materials. Nat. Mater. 14, 23-36 (2015).

50. Koffler, J. et al. Biomimetic 3D-printed scaffolds for spinal cord injury repair. Nat. Med. 25, 263-269 (2019).

51. Peng, M. et al. 3D printing of ultralight biomimetic hierarchical graphene materials with exceptional stiffness and resilience. Adv. Mater. 31, e1902930 (2019).

52. Kudo, A. \& Miseki, Y. Heterogeneous photocatalyst materials for water splitting. Chem. Soc. Rev. 38, 253-278 (2009).

53. Iwata, M., Teshima, M., Seki, T., Yoshioka, S. \& Takeoka, Y. Bio-inspired bright structurally colored colloidal amorphous array enhanced by controlling thickness and black background. Adv. Mater. 29, 1605050 (2017).

54. Reinhardt, $\mathrm{H}$. et al. Nanoscaled fractal superstructures via laser patterning-A versatile route to metallic hierarchical porous materials. Adv. Mater. Interfaces 2000253, 7 (2020).

55. Hu, F., Lyu, L. \& He, Y. A $3 D$ printed paper-based thermally driven soft robotic gripper inspired by cabbage. Int. J. Precis. Eng. Manuf. 20, 1915-1928 (2019).

56. Li, Y. et al. Bioinspired functional surfaces enabled by multiscale stereolithography. Adv. Mater. Technol. 4, 1800638 (2019).

57. Djumas, L., Molotnikov, A., Simon, G. P. \& Estrin, Y. Enhanced mechanical performance of bio-inspired hybrid structures utilising topological interlocking geometry. Sci. Rep. 6, 26706 (2016).

58. Sun, Z. et al. Fish-scale bio-inspired multifunctional ZnO nanostructures. NPG Asia Mater. 7, e232-e232 (2015).

59. Mirkhalaf, M., Dastjerdi, A. K. \& Barthelat, F. Overcoming the brittleness of glass through bio-inspiration and micro-architecture. Nat. Commun. 5, 3166 (2014).

60. Häsä, R. \& Pinho, S. T. Bio-inspired armour: CFRP with scales for perforation resistance. Mater. Lett. 273, 127966 (2020).

61. Qu, H., Yin, L., Ye, Y., Li, X., Liu, J. \& Feng, Y. et al. Bio-inspired stem-like composites based on highly aligned SiC nanowires. Chem. Engg J. 389 123466 (2020).

62. Liu, H., Huang, Y. \& Jiang, H. Artificial eye for scotopic vision with bioinspired all-optical photosensitivity enhancer. Proc. Natl Acad. Sci. USA 113, 3982-3985 (2016).

63. Karbalaei Akbari, M., Hu, J., Verpoort, F., Lu, H. \& Zhuiykov, S. Nanoscale alloxide-heterostructured bio-inspired optoresponsive nociceptor. Nano Micro Lett. 12, 83 (2020) 
64. Bharmoria, P. et al. Instantaneous fibrillation of egg white proteome with ionic liquid and macromolecular crowding. Commun. Mater. 1, 34 (2020).

65. Siéfert, E., Reyssat, E., Bico, J. \& Roman, B. Bio-inspired pneumatic shapemorphing elastomers. Nat. Mater. 18, 24-28 (2019).

66. Moreira, F. T. C., Truta, L. A. A. N. A. \& Sales, M. G. F. Biomimetic materials assembled on a photovoltaic cell as a novel biosensing approach to cancer biomarker detection. Sci. Rep. 8, 10205 (2018).

67. Estrada, S. \& Ossa, A. Nature-inspired protecto-flexible impact-tolerant materials. Adv. Eng. Mater. 22, 2000006 (2020).

68. Gantenbein, S. et al. Three-dimensional printing of hierarchical liquid-crystalpolymer structures. Nature 561, 226-230 (2018).

69. Pan, L. et al. A supertough electro-tendon based on spider silk composites. Nat. Commun. 11, 1332 (2020)
70. Tan, J., Jin, X. \& Chen, M. Bio-inspired synthesis of aqueous nanoapatite liquid crystals. Sci. Rep. 9, 466 (2019).

71. Zhao, Y. et al. Bio-inspired reversible underwater adhesive. Nat. Commun. 8, 2218 (2017).

72. Yang, S. et al. Ultra-antireflective synthetic brochosomes. Nat. Commun. 8 1285 (2017).

73. Otsuka, T., Fujikawa, S.-i, Yamane, H. \& Kobayashi, S. Green polymer chemistry: the biomimetic oxidative polymerization of cardanol for a synthetic approach to 'artificial urushi'. Polym. J. 49, 335-343 (2017).

74. Wani, O. M., Zeng, H. \& Priimagi, A. A light-driven artificial flytrap. Nat. Commun. 8, 15546 (2017). 